SECTION 1. Theoretical research in mathematics.

Sagat Zhunisbekov doctor of technical Sciences, Professor, academician of the National Engineering Academy of the Republic of Kazakhstan rector of the Taraz Technical Institute, Kazakhstan

\author{
Alexandr N. Shevtsov \\ candidate of Technical Sciences,
} President, Theoretical \& Applied Science, LLP associate Professor of the Department «Applied mathematics»,

Taraz State University named after M.H. Dulati, Kazakhstan

\title{
ALGORITHMS FOR CALCULATING THE PROPERTIES AND BEHAVIOR ANALYSIS OF FRACTIONAL-LINEAR MAPPINGS IN DELPHI.
}

In the article there are considered some issues of practical implementation on a computer fractional-linear functions of complex argument.

Key words: a complex function, Delphi, fractional-linear function, algorithm, circle.

\section{АЛГОРИТМЫ РАСЧЕТА СВОЙСТВ И АНАЛИЗ ПОВЕДЕНИЯ ДРОБНО-ЛИНЕЙНЫХ ОТОБРАЖЕНИЙ В СРЕДЕ DЕLРНІ.}

В статье рассмотренны некоторые вопросы практической реализации на компьютере дробно-линейных функций комплексного аргумента.

Ключевые слова: комплексная функция, дельфи, дробно-линейная функиия, алгоритм, окружность.

Исследование аналитических функций комплексного аргумента $w=f(z)=u(x, y)+i v(x, y)$, достаточно широко освещенно в курсе ТФКП (Теории функции комплексного переменного), но при этом зачастую возникают проблемы наглядности, в процессе изучения, а также практические методы компьютерной реализации.

Как известно [1-2], в тех точках $z_{0} \in G$, где $f^{\prime}\left(z_{0}\right) \neq 0$, функция $w=f(z)$ обладает так называемым свойством конформности. Трудности изучения этих свойств связаны, в первую очередь, с особенностями 
изучаемого объекта. Довольно сложно представить поведение функции комплексного переменного наглядно[3].

Рассмотрим дробно-линейную функцию

$$
w=f(z)=\frac{a z+b}{c z+d}
$$

где $a, b, c, d$ - постоянные комплексные числа, причем $c, d$ одновременно не равны нулю. Первая производная от $f(z)$ примет вид:

$$
f^{\prime}(z)=\frac{a d-c b}{(c z+d)^{2}}
$$

при соблюдении условия $a d-b c \neq 0$, отображение осуществляемое дробнолинейной функцией, конформно и взаимно-однозначно в области $G$, получаемой из расширенной комплексной плоскости исключением точек $z^{\prime}=\infty$ и $z^{\prime}=-\frac{d}{c}$.

При рассмотрении примеров [3, с.12], находят образы окружностей:

$$
\begin{gathered}
|z+i|=\frac{1}{2} \text { при } w=\frac{z-i}{2 z+i}, \\
|z|=1 \text { при } w=\frac{z-i}{2 z+i} .
\end{gathered}
$$

Разработаем алгоритмы построения этих отображений в среде Delphi в реальном времени [4], т.е. пользователь сможет наблюдать не только результат построения, а также и видеть сам процесс (часть типичных подпрограмм, для создания отчетов, в коде опущенна).

unit Unit1;

interface

uses

Windows, Messages, SysUtils, Variants, Classes, Graphics, Controls, Forms, Dialogs, Grids, StdCtrls, Buttons, ComCtrls, Menus, TeEngine, Series, ExtCtrls, TeeProcs, Chart, CheckLst, Spin,FileCtrl, Clipbrd, Office_Tlb,Word_TLB,ComObj,Math;

type

TForm $1=\operatorname{class}($ TForm $)$

PageControl1: TPageControl; 
MainMenu1: TMainMenu;

N1: TMenuItem;

N2: TMenuItem;

N3: TMenuItem;

N4: TMenuItem;

N5: TMenuItem;

TabSheet1: TTabSheet;

BitBtn1: TBitBtn;

FileListBox1: TFileListBox;

Memo1: TMemo;

StringGrid1: TStringGrid;

TabSheet2: TTabSheet;

BitBtn2: TBitBtn;

Label1: TLabel;

RadioGroup1: TRadioGroup;

StringGrid2: TStringGrid;

Image 1: TImage;

Image 2: TImage;

Panel1: TPanel;

BitBtn3: TBitBtn;

Memo2: TMemo;

Memo3: TMemo;

Memo4: TMemo;

ProgressBar1: TProgressBar; procedure FileListBox1Click(Sender: TObject);

procedure N5Click(Sender: TObject);

procedure N2Click(Sender: TObject);

procedure BitBtn1Click(Sender: TObject);

procedure BitBtn2Click(Sender: TObject);

procedure FormCreate(Sender: TObject);

procedure RadioGroup1Click(Sender: TObject);

procedure BitBtn3Click(Sender: TObject);

private

\{ Private declarations \}

WordApp:OleVariant;

WordTables,WordTables 1:OleVariant;

Procedure WordAppExcept(Sender:TObject; E:Exception);

public

\{Public declarations \}

end;

var

Form1: TForm1; 
nn,zz:integer;

implementation

$\{\$ \mathrm{R} * \mathrm{dfm}\}$

procedure TForm1.BitBtn3Click(Sender: TObject);

begin

image 1.Picture.Bitmap.SaveToFile('рисунок-.bmp');

image2.Picture.Bitmap.SaveToFile('oбраз-.bmp');

end;

procedure TForm1.FileListBox1Click(Sender: TObject);

var i,j:integer; t:real;

begin

memo1.Clear; memo1.Lines.LoadFromFile(FileListBox1.FileName);

for $\mathrm{I}:=0$ to memo1.Lines.Count -1 do

begin

//StringGrid1.Cells[0,i+1]:=inttostr(i+1);//GetToken(memo1.Lines.Strings[i],' ',1);

$\mathrm{j}:=1$;

while GetToken(memo1.Lines.Strings[i],' ',j)<>" do

begin

StringGrid1.Cells[j,i+1]:=GetToken(memo1.Lines.Strings[i],' ',j);

$\mathrm{j}:=\mathrm{j}+1$;

//StringGrid1.Cells[2,i+1]:=GetToken(memo1.Lines.Strings[i], ',2);

end;

end;

stringgrid1.RowCount: $=$ memo1.Lines.Count +2 ;

end;

function LoadTable(s:tmemo \{string $\}$;StringGrid:TStringGrid):boolean;

var $\mathrm{i}, \mathrm{j}:$ integer;

begin

form1.memo1.Clear;

form1.memo1.Text:=s.Text;/.Lines.LoadFromFile(s);

form1.label1.Caption:=form1.memo1.Lines.Strings[0];

for $\mathrm{I}:=1$ to form1.memo1.Lines.Count $-1 \mathrm{do}$

begin

$\mathrm{j}:=1$;

while GetToken(form1.memo1.Lines.Strings[i],'=',j) $<>$ do begin

StringGrid.Cells[j-1,i-1]:=GetToken(form1.memo1.Lines.Strings[i],'=',j); $\mathrm{j}:=\mathrm{j}+1$; 
end;

end;

stringgrid.RowCount:=form1.memo1.Lines.Count-1;

$\mathrm{nn}:=$ stringgrid.RowCount-1;

end;

procedure TForm1.FormCreate(Sender: TObject);

begin

RadioGroup1.ItemIndex: $=0$;

LoadTable(memo2 \{'Данные-0.txt'\},StringGrid1);

LoadTable(memo4\{'Данные-2.txt'\},StringGrid2);

end;

function fnMod(x,y:real):real;

begin

result:=power( $\mathrm{x} * \mathrm{x}+\mathrm{y} * \mathrm{y}, 0.5)$;

end;

function fnArg (x,y:real):real;

var arg:real;

begin

IF $\mathrm{x}<>0$ THEN

begin

$$
\begin{aligned}
& \arg :=\operatorname{ArcTaN}(\mathrm{y} / \mathrm{x}) ; \\
& \text { IF } \arg <0 \text { THEN } \arg :=\arg +\text { pi } \\
& \text { ELSE } \\
& \text { IF } \mathrm{y}>=0 \text { THEN } \arg :=\text { pi } / 2 \text { ELSE } \arg :=- \text { pi } / 2 \text {; }
\end{aligned}
$$

END;

end;

function fnRe(x1,y1,x2, y2:real):real;

begin

result:=x1*x2-y1*y2;

end;

function fnIm(x1,y1,x2, y2:real):real;

begin

result: $=\mathrm{x} 1 * \mathrm{y} 2+\mathrm{y} 1 * \mathrm{x} 2$;

end;

function fnU (x,y,L,a, Re:real):real;

begin 
result:= $L^{*}\left(x^{*} \operatorname{COS}(a)-y^{*} \operatorname{SIN}(a)\right)+R E$;

end;

function fnV (x,y,L,a, Im:real):real;

begin

result: $=\mathrm{L} *\left(x^{*} \operatorname{SIN}(\mathrm{a})+\mathrm{y} * \operatorname{COS}(\mathrm{a})\right)+\operatorname{Im}$;

end;

function Koord(image:timage;minx,maxx,miny,maxy:integer):boolean;

var $i, j:$ integer;

begin

image.Canvas.Pen.Width: $=1$;

image.Canvas.Brush.Color: $=$ clwhite;

image.Canvas.FillRect(rect $(0,0,10000,10000))$;

FOR j: $=-20$ TO 20 do

begin

image.Canvas.MoveTo(250+trunc $\left.\left(z z^{*} \min X\right), 250-z^{*} \mathrm{j}\right)$;

image.Canvas.LineTo(250+trunc(zz*maxX), 250-zz*j);

image.Canvas.MoveTo(250+zz*j, 250-trunc $\left(\mathrm{zz}^{*} \min \mathrm{Y}\right)$ );

image.Canvas.LineTo(250+zz*j,250-trunc(zz*maxY));

image.Canvas. TextOut(250+zz*j-12,255, inttostr(j));

image.Canvas.TextOut(250-12,250-zz*j+5, inttostr(j));

end;

image.Canvas.Pen.Width:=3;

image.Canvas.MoveTo( 0,250$)$;

image.Canvas.LineTo(1900,250);

image.Canvas.MoveTo(250,0);

image.Canvas.LineTo(250,1900);

image.Canvas.Pen.Width:=1;

end;

procedure TForm1.BitBtn1Click(Sender: TObject); // Расчет

var

$\min X, \max X, \min Y, \max Y, I, j: I n t e g e r ;$

s, modc,Rea1,Ima1,Reb1,Imb1,Rea2,Ima2,Reb2,Imb2,

moda1, arga1,moda2, arga2,modd,p,

Re1,Im1,Re2,Im2,Re3,Im3,

R,x0,y0,Rea,Ima,Reb,Imb,Rec,Imc,Red,Imd,

u1,v1,u,v,t,h,k, x,y,a,m,n:Real; // Задаем переменные

label 2;

begin 


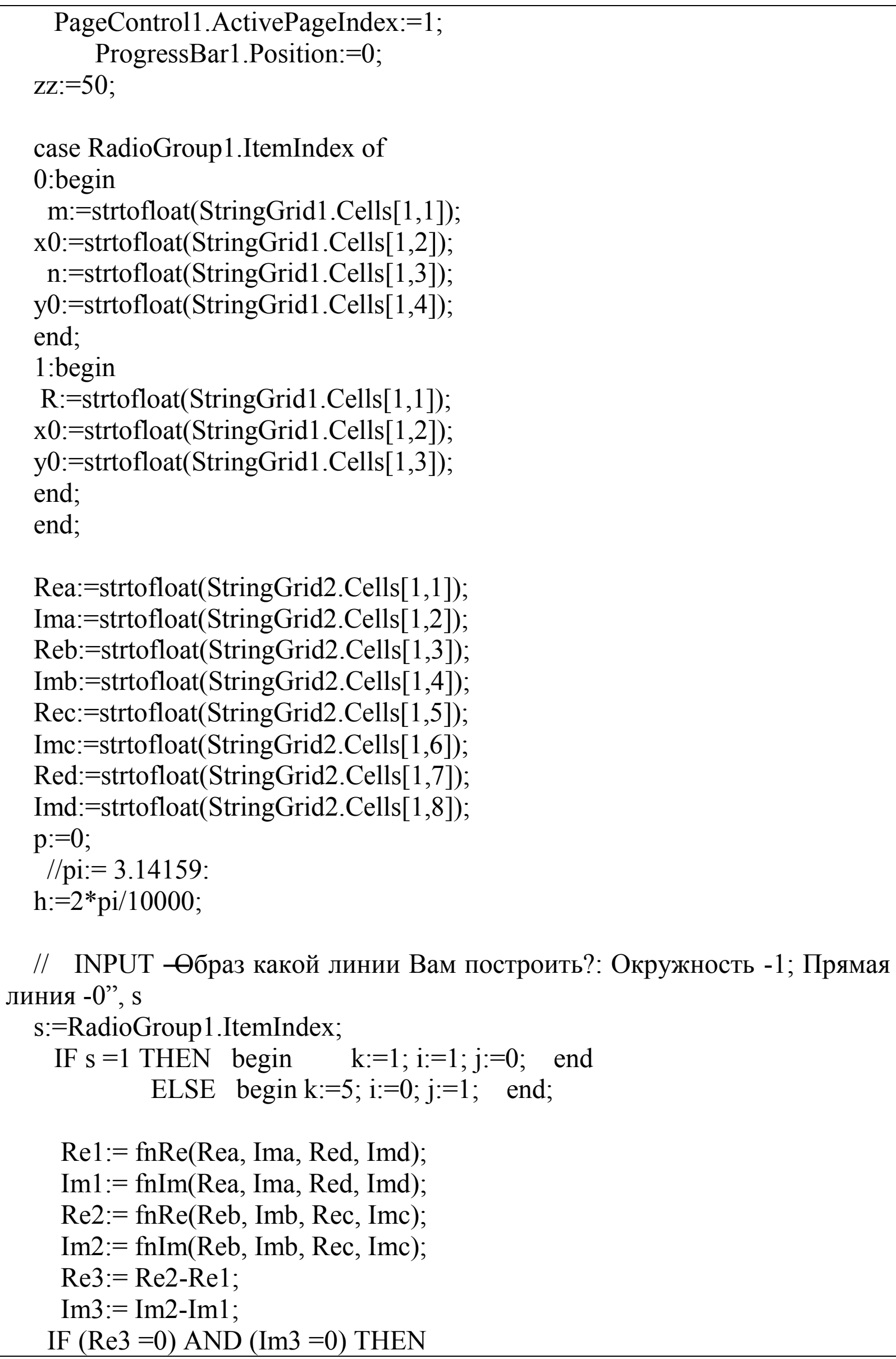


begin

showmessage('Данные не верны! Введите заново!');

// exit;

end;

IF $(\operatorname{Rec}=0)$ AND $(\operatorname{Imc}=0)$ THEN

begin

modd:= power(fnMod(Red, Imd),2);

Rea1:= fnRe(Rea, Ima, Red, -Imd)/modd;

Ima $1:=$ fnIm(Rea, Ima, Red, -Imd)/modd;

Reb1:= fnRe(Reb, Imb, Red, -Imd)/modd;

$\operatorname{Imb} 1:=$ fnIm(Reb, Imb, Red, -Imd)/modd;

end

ELSE

begin

modc:= power(fnMod (Rec, Imc),2);

$\mathrm{p}:=1$;

Rea1:= Rec;

Ima $1:=\operatorname{Imc}$;

Reb1:= Red;

$\operatorname{Imb} 1:=\operatorname{Imd} ;$

Rea2:= fnRe(Re3, Im3, Rec, -Imc)/modc;

$\operatorname{Ima} 2:=$ fnIm(Re3, Im3, Rec, $-\operatorname{Imc}) /$ modc;

$\operatorname{Reb} 2:=$ fnRe(Rea, Ima, Rec, -Imc)/modc;

$\operatorname{Imb} 2:=$ fnIm(Rea, Ima, Rec, - Imc)/modc;

end;

moda1:= fnMod (Rea1, Ima1);

argal:= fnArg(Reb1, Imb1);

moda2:= fnMod (Rea2, Ima2);

$\operatorname{arga2}:=$ fnArg(Reb2, Imb2);

$\min X:=-9$;

$\max X:=9$;

$\min Y:=-9$;

$\max Y:=9$;

Koord(image1,minx,maxx,miny,maxy);

Koord(image 2,minx,maxx,miny,maxy);

image1.Canvas.Brush.Color:=clred;

image1.Canvas.Pen.Width:=3;

image2.Canvas.Brush.Color:=clred; 
image2.Canvas.Pen.Width:=3;

$\mathrm{t}:=-\mathrm{k}^{*} \mathrm{pi}$

while $\mathrm{t}<\mathrm{k}^{*}$ pi do

begin

$\mathrm{x}:=\mathrm{x} 0+\mathrm{i}^{*} \mathrm{R} * \cos (\mathrm{t})+\mathrm{j} * \mathrm{~m} * \mathrm{t}$;

$\mathrm{y}:=\mathrm{y} 0+\mathrm{i}^{*} \mathrm{R} * \operatorname{SIN}(\mathrm{t})+\mathrm{j}^{*} \mathrm{n} * \mathrm{t}$;

$\mathrm{u}:=\mathrm{fnU}(\mathrm{x}, \mathrm{y}$, modal, arga1, Reb1);

$\mathrm{v}:=\operatorname{fnV}(\mathrm{x}, \mathrm{y}$, modal, arga1, Imb1);

IF $\mathrm{p}<>1$ THEN goto 2 ;

$\mathrm{s}:=\mathrm{u}^{*} \mathrm{u}+\mathrm{v}^{*} \mathrm{v}$;

$\mathrm{u} 1:=\mathrm{u} / \mathrm{s}$;

$\mathrm{v} 1:=\mathrm{v} / \mathrm{s}$

$\mathrm{u}:=\mathrm{fnU}(\mathrm{u} 1, \mathrm{v} 1$, moda2, arga2, Reb2);

$\mathrm{v}:=\mathrm{fnV}(\mathrm{u} 1, \mathrm{v} 1$, moda2, arga $2, \operatorname{Imb} 2)$;

2:

image 1.Canvas.FillRect $\left(\operatorname{rect}\left(250+\operatorname{trunc}\left(\mathrm{zz}^{*} \mathrm{x}\right)-2,250-\operatorname{trunc}\left(\mathrm{zz}^{*} \mathrm{y}\right)-\right.\right.$

$2,250+\operatorname{trunc}(\mathrm{zz} * \mathrm{x})+2,250-\operatorname{trunc}(\mathrm{zz} * \mathrm{y})+2))$;

image2.Canvas.FillRect(rect $\left(250+\operatorname{trunc}\left(\mathrm{zz}^{*} \mathrm{u}\right)-2,250-\operatorname{trunc}\left(\mathrm{zz}^{*} \mathrm{v}\right)-\right.$

$\left.\left.2,250+\operatorname{trunc}(\mathrm{zz} * \mathrm{u})+2,250-\operatorname{trunc}\left(\mathrm{zz}^{*} \mathrm{v}\right)+2\right)\right)$;

sleep(1);

application.ProcessMessages;

image1.Update;

image2.Update;

ProgressBar1.Position:=trunc(int $(\mathrm{t}))+15$;

$\mathrm{t}:=\mathrm{t}+\mathrm{h}$;

end;

end;

procedure TForm1.N2Click(Sender: TObject);

begin

application.Terminate;

end;

procedure TForm1.N5Click(Sender: TObject);

begin

showmessage('Автор: к.т.н. Шевцов А.Н.');

end;

procedure TForm1.RadioGroup1Click(Sender: TObject);

begin

case RadioGroup1.ItemIndex of 
0:LoadTable(memo2 \{'Данные-0.txt'\},StringGrid1);

1:LoadTable(memo3 \{'Данные-1.txt'\},StringGrid1); end;

LoadTable(memo4 \{'Данные-2.txt'\},StringGrid2); end;

end.

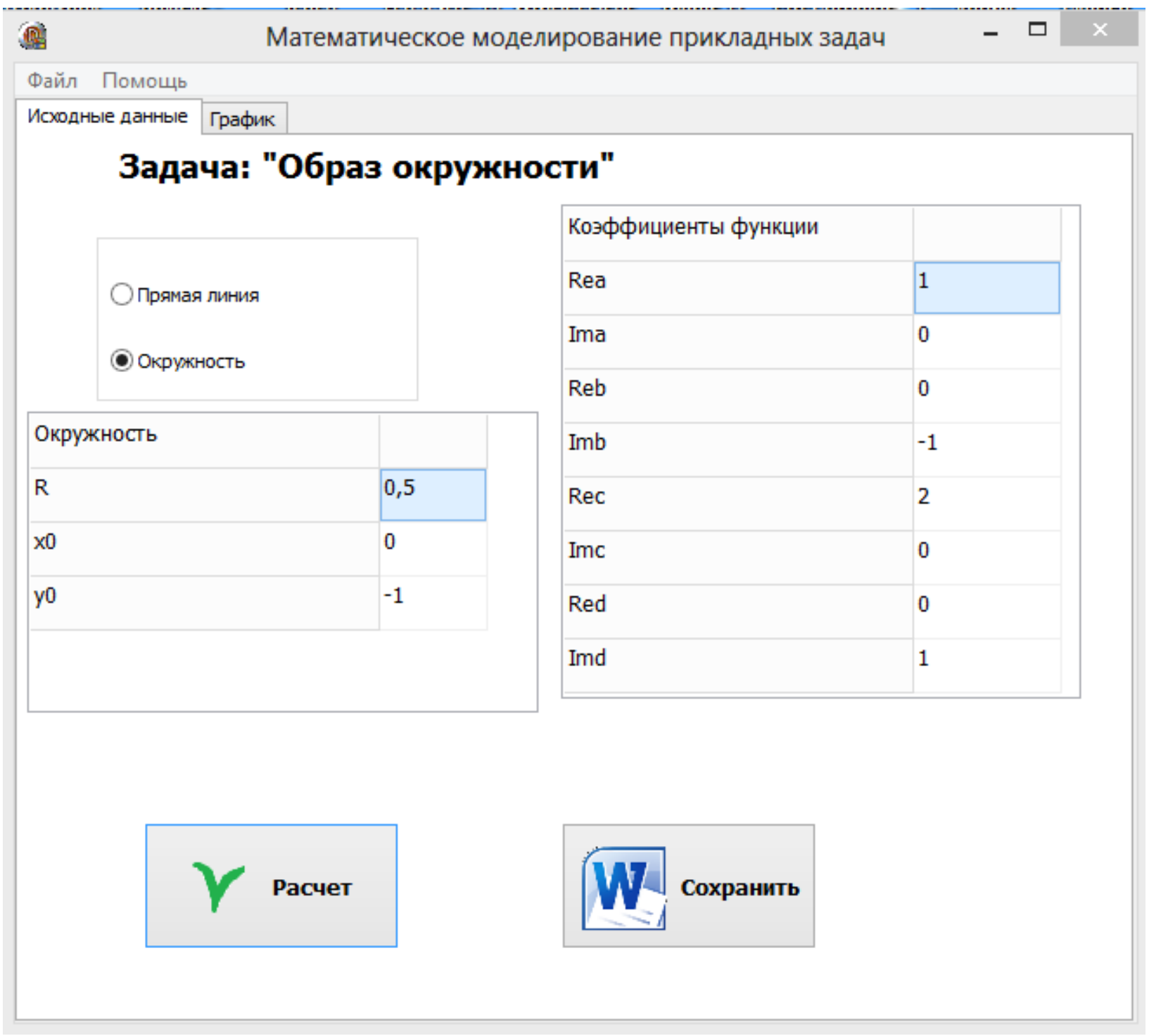

Рисунок 1 - Окно программы. 
Математическое моделирование прикладных задач

$-\square$

Исходые данные График
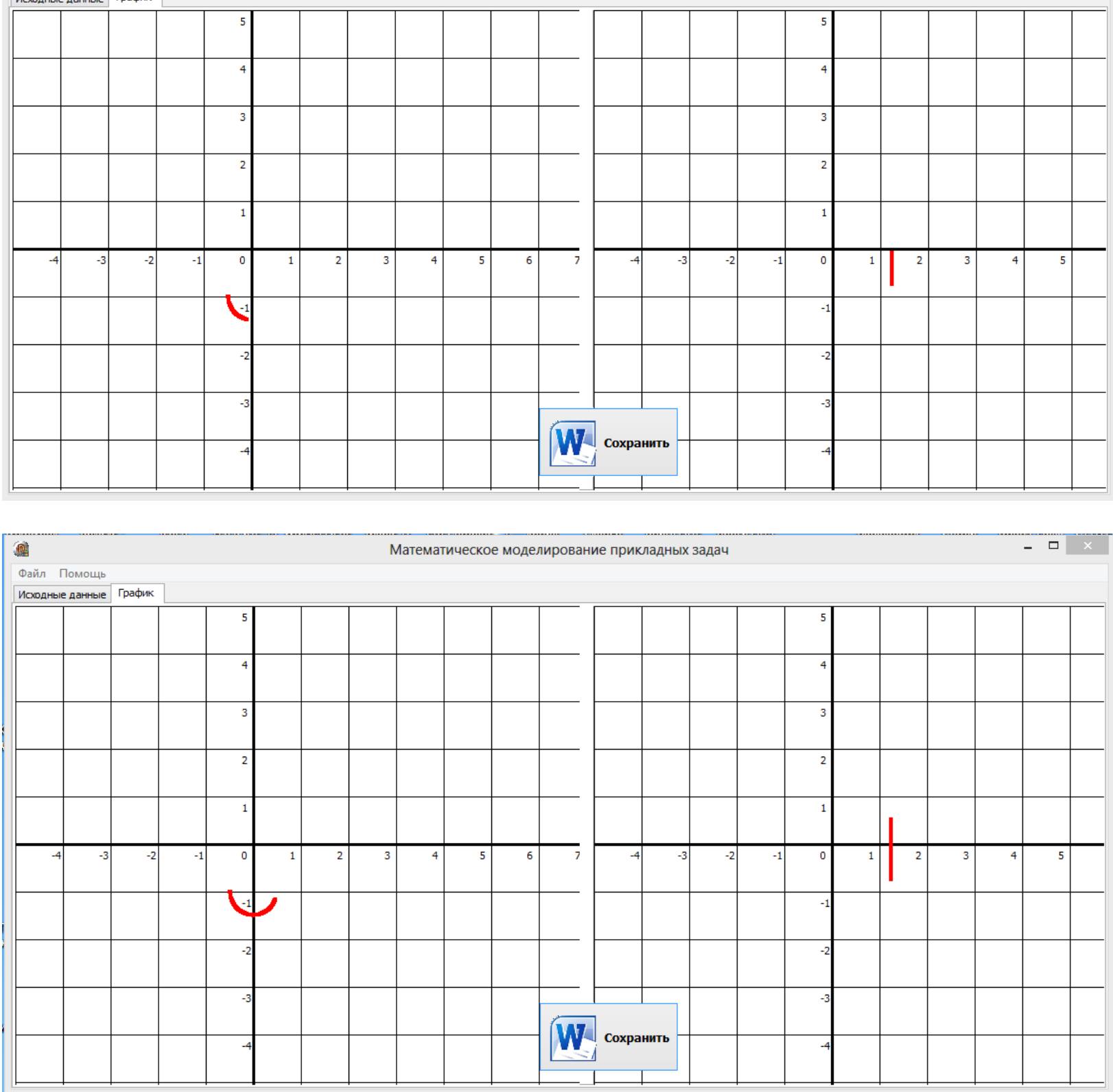

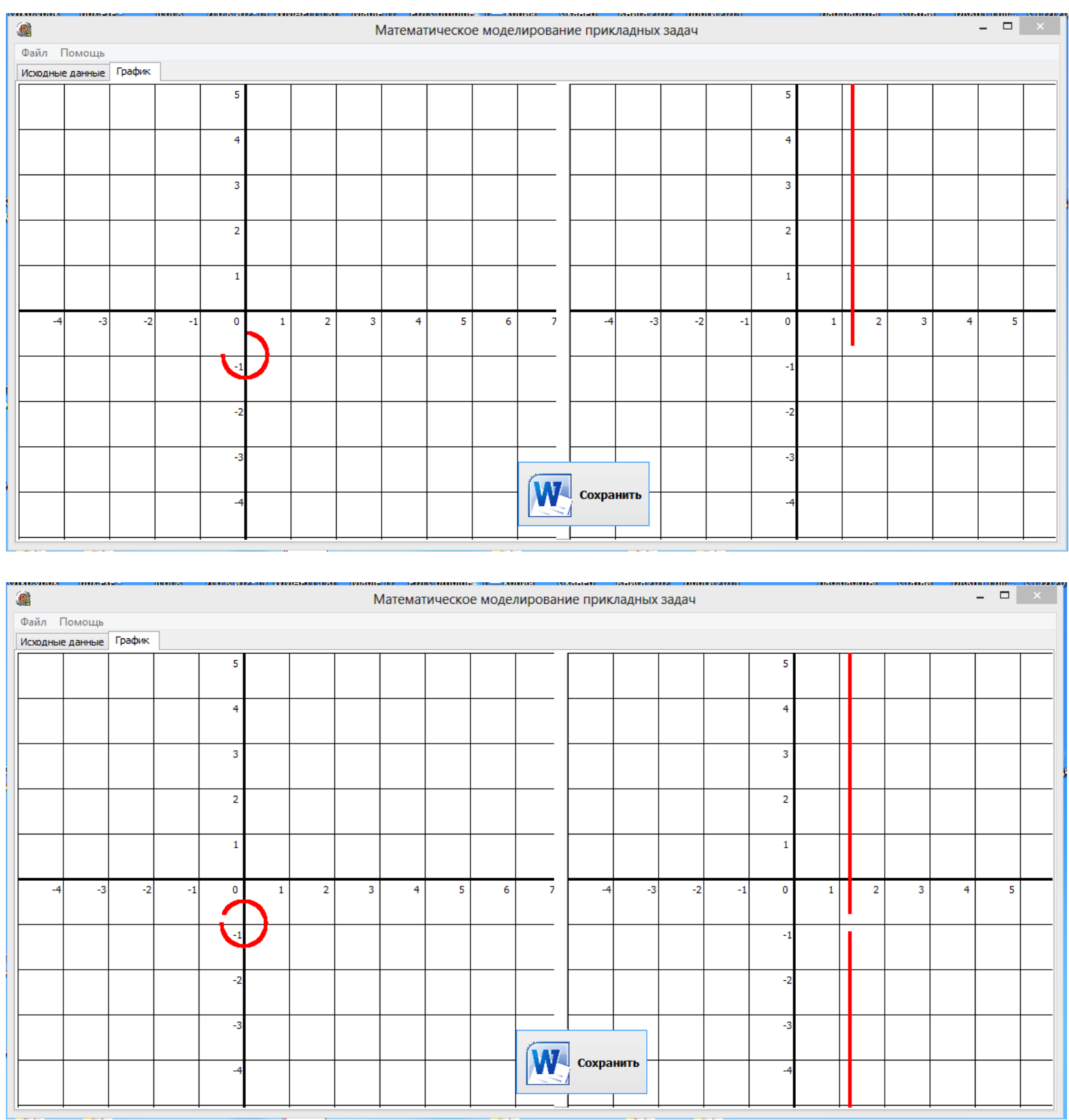


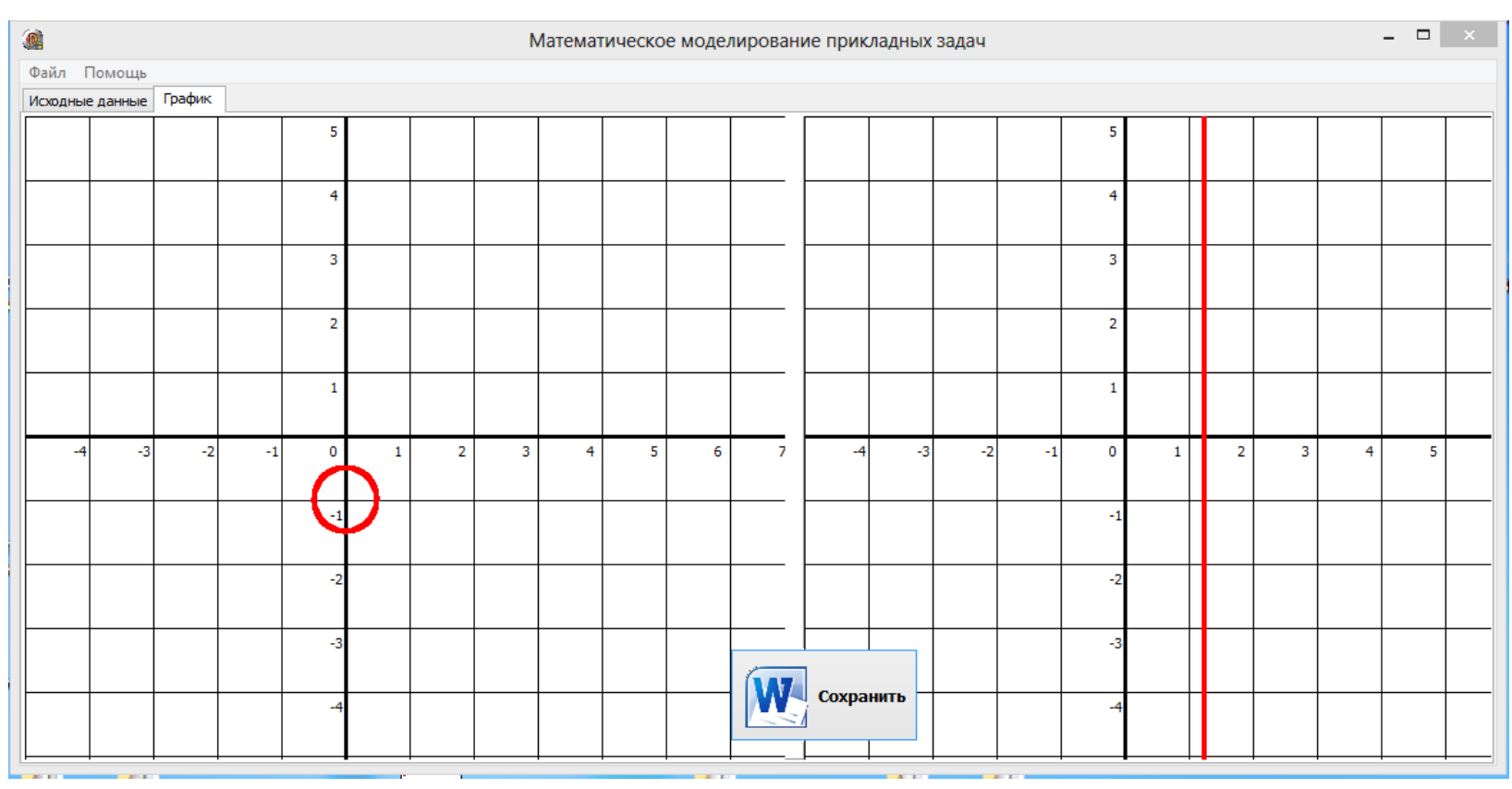

Рисунок 2 - Процесс построения отображения.

Разработанные алгоритмы и программа позволяют решать и исследовать целый ряд дробно-линейных отображений, при различных начальных условия и вводимых ограничениях.

\section{Литература}

1. Маркушевич А.И. Введение в теорию аналитических функций. - М.: Просвещение, 1977.-320с.

2. Привалов И.И. Введение в теорию функций комплексного переменного. -М.: Наука, 1977.-444c.

3. Абиев Н.А., Чанбаева А.И. Интерпретация свойств дробно-линейных отображений в среде Delphi. - Проблемы естественно-математического образования в исследованиях профессионально ориентированной личности: материалы пятой международной научно-практической конференции молодых ученых, студентов, аспирантов: 14-15 апреля 2012г.:в 2ч. Ч.1/ ФГБОУ ВПО «СГПИ». - Соликамск: СГПИ, 2012. С.1113.

4. Nevzorov V. Delphi Russian Knowledge Base. -Chicago, USA. -2007, more than $4000 \mathrm{p}$. 